\title{
El apoyo al estudio independiente en un curso de comprensión de lectura con "Hot Potatoes"
}

\author{
JuAN PABLo ZÚÑIGa Vargas \\ Escuela de Lenguas Modernas \\ Universidad de Costa Rica
}

\begin{abstract}
Resumen
Este artículo describe el diseño y la puesta en práctica de una estrategia didáctica para apoyar el estudio independiente en un grupo de estudiantes de comprensión de lectura en inglés durante un semestre en la Universidad de Costa Rica. Para este fin, se diseñaron diez pruebas de práctica interactivas para el estudiantado con la herramienta de autor "Hot Potoatoes." Estas pruebas se pusieron a disposición de los estudiantes en una página web personal del docente con al menos una semana de antelación a cada evaluación formal del curso. Después de esto, se administró un cuestionario con dos preguntas cerradas y tres preguntas abiertas para que los estudiantes valoraran esta experiencia. Los resultados obtenidos señalan que las principales ventajas para el apoyo al estudio independiente son la preparación efectiva para las evaluaciones del curso, la correspondencia entre el contenido impartido y el evaluado, el reforzamiento de conceptos y la facilidad del trabajo virtual.
\end{abstract}

Palabras claves: estudio independiente, auto-evaluación, herramientas de autor, comprensión de lectura, Hot Potatoes

\begin{abstract}
This article describes the design and implementation of a didactic strategy intended to promote students' independent study in a group of reading comprehension in English during one semester at the University of Costa Rica. For this purpose, ten interactive practice tests were designed for the students using the authoring tool called "Hot Potatoes." These tests were made available for the students on a personal web page owned by the teacher at least one week prior to each formal evaluation of the course. After this, a questionnaire was administered to the students for them to assess this experience. The obtained results indicate the main advantages to support independent study are the effective preparation for the evaluations of the course, the correspondence between the content taught and evaluated, the reinforcement of concepts, and the ease of virtual work.
\end{abstract}

Key words: independent study, self-evaluation, authoring tools, reading comprehension, Hot Potatoes 


\section{Introducción}

$\mathrm{E}$ ste artículo describe el diseño y puesta en práctica de una estrategia didáctica, en la cual se utilizó la herramienta de autor "Hot Potatoes" para la creación de pruebas de práctica para grupo de estudiantes del curso LM-1030, Estrategias de Lectura en Inglés I durante el segundo semestre de 2013, en la Escuela de Lenguas Modernas de la Universidad de Costa Rica. Las pruebas tuvieron el fin de complementar la materia vista en clase con práctica y, por ende, de apoyar a los estudiantes en sus horas de estudio independiente. Las prácticas fueron puestas a disposición de los estudiantes en una página web personal del docente con anterioridad a las evaluaciones formales del curso. A continuación, se describirán las situaciones que motivaron al docente a diseñar esta estrategia didáctica, los pasos que se siguieron para su formulación y puesta en práctica y los resultados obtenidos en esta experiencia.

\section{Justificación}

El curso LM-1030, Estrategias de Lectura en Inglés I, al ser un curso de servicio, no posee ningún requisito ni correquisito para ser matriculado. Por esta razón, los docentes que lo imparten deben atender a una población estudiantil muy diversa, tanto por la carrera que cursan como por el nivel de la carrera en el que se encuentran y el nivel de inglés que poseen. En el curso, las horas semanales se distribuyen en seis horas de trabajo presencial y seis horas de trabajo extraclase. En particular, el curso LM-1030 se caracteriza por ser eminentemente práctico. En él se desarrollan diversas estrategias de lectura orientadas al trabajo con textos en lengua inglesa y, si bien es cierto que los estudiantes deben estar familiarizados con los fundamentos teóricos de las estrategias de lectura por emplear en el curso, el simple estudio de la teoría no es de gran ayuda para alcanzar los objetivos planteados en su programa.

En un curso de estrategias de lectura, el estudio independiente es crucial. Un estudiante de LM-1030 no debe simplemente conformarse con la preparación que se le brinda en clase y conformarse con repasar los aspectos teóricos y prácticos presentes en la antología. Idealmente, cada estudiante, en sus horas de trabajo extraclase, debería buscar textos en inglés para leerlos y poner en práctica las estrategias que se estudian en clase. A criterio del autor de este artículo, esto siempre ha representado un gran desafío, ya que en la actualidad muchos estudiantes no poseen buenos hábitos de lectura en su lengua materna, ni han desarrollado efectivamente sus estrategias de lectura. Por consiguiente, no se puede esperar que tengan un desempeño satisfactorio en un curso de lectura en una lengua extranjera si tampoco son buenos lectores en su lengua materna. Además, por experiencias previas desfavorables con el inglés, muchos estudiantes no se sienten motivados para estudiar este idioma. Sumado a esto, una inquietud constante por parte de ellos es que no tienen una idea clara de cómo se estructura una evaluación en el curso, ya que en él se presentan y se 
ponen en práctica las estrategias de lectura una a una (de manera segregada), y la antología (a criterio de los estudiantes) no incluye muchos ejemplos de pruebas de práctica donde se integren diversas estrategias para abordar un mismo texto.

En respuesta a las situaciones antes descritas, el docente optó por el uso de la herramienta de autor "Hot Potatoes" para la creación de pruebas de práctica para sus estudiantes, las cuales estarían disponibles en un sitio web personal con al menos una semana de antelación a todas las pruebas a realizarse durante el semestre. Estas prácticas se adaptaron de pruebas cortas y exámenes que el docente aplicó en años anteriores en su curso, con el propósito de proveer a los estudiantes de material de práctica adicional que pudieran utilizar en sus horas de estudio individual (trabajo extraclase) y que también integrara diferentes estrategias de lectura para analizar un mismo texto. Es importante señalar en este momento, que el proyecto no tuvo la intención de que el curso se convierta en un espacio donde meramente se aprendiera la habilidad de aprobarlo. Todas las estrategias estudiadas se orientaron a alcanzar la meta final de guiar al estudiante para que desarrollara sus destrezas metacognitvas y se volviera un lector más crítico y más eficiente al leer en inglés y en su lengua materna. Por consiguiente, se planteó la siguiente pregunta generadora para el desarrollo del proyecto:

¿Es posible apoyar el estudio independiente de los estudiantes del curso LM-1030 por medio del uso de pruebas de práctica diseñadas con la herramienta "Hot Potatoes" y, de ser así, qué ventajas les ofrece a los estudiantes?

\section{Fundamentación teórica}

\section{El uso del software de autor en la enseñanza: Lo innovador más lo tradicional}

El software de autor comprende diferentes tipos de programas informáticos que permiten la creación de materiales didácticos digitales a partir de plantillas prediseñadas, en las cuales se puede insertar texto, imágenes y sonido (Martín, 2004). Estos materiales pueden ser compartidos por medio de discos compactos (CD-ROM) o dispositivos de almacenamiento USB, ser subidos a páginas web como archivos adjuntos (Dudeney y Hockly, 2008) o incluso ser compartidos con los estudiantes por medio de correo electrónico, ya que la mayoría de las herramientas de autor convierten los archivos al formato HTML, el cual técnicamente se puede abrir en cualquier computadora con sólo tener un navegador de Internet y sin limitaciones de sistemas operativos. En sí, la distribución de estos materiales es muy versátil y simple, lo cual facilita que más personas puedan utilizarlos.

Las plantillas de diseño antes mencionadas sirven para crear actividades, tales como asocies, crucigramas, ejercicios de selección única, respuesta corta, textos con palabras faltantes para completar, entre otros. A primera vista, este tipo de actividades puede no constituir nada nuevo. No obstante, es el medio utilizado lo que es innovador. Las herramientas de autor proveen al estudiante 
de realimentación inmediata y, a diferencia de los materiales impresos, permiten que las actividades se realicen varias veces hasta que el estudiante se sienta complacido con su desempeño (Zúñiga y Seravalli, 2014), sin tener que borrar lo que se escribió para comenzar de nuevo.

En este sentido, en el siglo XXI, es necesario que los docentes mediten sobre sus estrategias de mediación pedagógica y las adapten a las nuevas necesidades de los estudiantes (Níkleva y López, 2012) y a las posibilidades que ofrecen las tecnologías de información y comunicación emergentes. Este desafío supone ser crítico a la hora de valorar las alternativas tecnológicas que se puedan utilizar para un fin específico y adoptar aquellas que demuestren una utilidad verdadera más allá de ser algo diferente; las herramientas de autor, empleadas correctamente, sirven muy bien a este propósito.

\section{"Hot Potatoes"}

Actualmente existe una gran variedad de herramientas de autor; "Hot Potatoes," a criterio de este docente, es una de las más versátiles. Es un programa gratuito que permite diseñar actividades interactivas en formato de página web para los estudiantes sin presuponer ningún conocimiento especializado en informática (Half-Baked Software Inc., 2009). El trabajo con esta herramienta es sumamente sencillo y flexible, lo cual facilita la creación materiales en poco tiempo y le permite al docente concentrarse en algo más importante, los contenidos a reforzar por medio de este recurso. Además, Hot "Potatoes" se puede instalar en computadoras con distintos sistemas operativos, tales como Windows, Mac (Dudeney y Hockly, 2008) o incluso en Linux por medio del programa de compatibilidad "Wine" (Half-Baked Software Inc., 2013).

\section{La promoción del estudio independiente en los estudiantes}

De acuerdo con Frei, Gammill e Irons (2007), con el uso de recursos tecnológicos, los estudiantes (y no solo el docente) deberían ser partícipes de la evaluación. En la actualidad, los docentes, idealmente, deberían estar formando estudiantes más críticos y que asuman una mayor responsabilidad por su propio aprendizaje, por lo que, para un mejor desempeño en cualquier programa de estudio, es necesario que los estudiantes organicen su tiempo para contar con suficientes momentos de estudio y práctica individual. Concretamente, contar con materiales de práctica adicionales en Internet podría dar respuesta a esta necesidad ya que, como señala Martín (2004):

El trabajo en la red tiene la ventaja de que permite un alto grado de autonomía al alumno, no sólo a nivel organizativo, sino también en cuanto al modo en que aprende, lo cual a su vez está determinado por los patrones predominantes en su personal estilo de aprendizaje. (p. 108) 
Es posible concluir entonces que los materiales interactivos disponibles en Internet tienen el potencial de estimular positivamente al estudiante a la hora de practicar contenidos vistos en clase en sus horas de estudio independiente. Cabe mencionar también que esta práctica puede realizarse en el instante que el estudiante considere más conveniente, en diferentes momentos, no necesariamente de una manera lineal (Níkleva y López, 2012) y realizar los ejercicios cuantas veces se desee (Erben, Ban, y Castañeda, 2009). Asimismo, al utilizar materiales que brindan realimentación automática e inmediata, el estudiante autoevalúa y modera su proceso de aprendizaje y por ende aprovecha mejor sus momentos de estudio.

Díaz y Muriá (1998) señalan que, en la promoción del estudio independiente, el papel del docente es crucial y resaltan "que el alumno no construye el conocimiento en solitario" (p. 22) y más adelante explican que "la formación en habilidades cognoscitivas ... se ha concentrado casi exclusivamente en el alumno, olvidando que el docente funge como mediador del encuentro entre el estudiante y el conocimiento" (p. 22). Es recomendable entonces que el docente brinde a sus estudiantes recursos adicionales de práctica que complementen los temas vistos en clase. Así pues, el docente puede ser un mediador en el proceso de estudio independiente de sus estudiantes por medio del diseño de materiales o pruebas de práctica que reflejen los contenidos y competencias que se espera que desarrollen los estudiantes.

Existen diferentes autores que abordan el estudio independiente exclusivamente en el contexto de la educación a distancia. No obstante, las ideas y principios descritos para este ámbito educativo son totalmente aplicables en otras modalidades de la educación superior. Galarza (1996) se refiere en este sentido a que "los estudiantes están acostumbrados a aprender pasivamente, de manera acrítica y, principalmente, por medio de la memorización" (p. 3) así como Ortiz (2011) señala que "las personas que toman la iniciativa en el autoaprendizaje, tienen más posibilidades de retener lo que aprenden que el estudiante pasivo" (p. 2). Dichos postulados suponen un cambio radical y necesario en el sistema educativo. En la actualidad, mucho se habla sobre promover la autonomía del estudiante, pero tales sugerencias pueden ser meramente teóricas e incluso románticas, donde no se va más allá de señalar aquello que no está bien y de describir vagamente cómo mejorarlo sin llegar a propuestas concretas y tangibles de cómo hacerlo. Nuevamente, la idea del autor de este artículo es simple y puntual: proveer a los estudiantes de prácticas digitales e interactivas que sirvan de apoyo en sus horas de estudio individual.

Martínez, Martínez, Moyotl, Murguia y Oliveros (2013), en relación con el estudio independiente, mencionan que el docente debe estar familiarizado con recursos tecnológicos que propicien en sus estudiantes "la construcción de su propio conocimiento" (párr. 11). No quiere decir que la tecnología por sí misma promueva la construcción del conocimiento. Es necesario recordar que la tecnología es simplemente un medio para lograr tal fin; los docentes deben ser críticos a la hora de juzgar las posibilidades pedagógicas y didácticas que ofrecen diferentes recursos tecnológicos y adoptar aquellos que realmente traigan beneficios 
al proceso de aprendizaje de los estudiantes. En particular y, a criterio de este docente, la herramienta "Hot Potatoes" posee muchas características deseables para este propósito, como se ha mencionado con anterioridad. Es posible añadir que, con esta herramienta, se puede incluir casi cualquier contenido de cualquier materia y adaptarlo según las características de las diferentes plantillas de diseño y hacer que refleje la manera de plantear los ejercicios en las prácticas y evaluaciones de un determinado curso.

A propósito del estudio independiente, Dunlosky, Rawson, Marsh, Nathan y Willingham (2013) llevaron a cabo una amplia revisión de la literatura sobre diez técnicas de aprendizaje individual comúnmente empleadas por estudiantes y valoraron el nivel de efectividad de cada una. De todas, realizar pruebas de práctica resultó estar entre las técnicas más útiles; se concluyó que el uso de pruebas de práctica o evaluaciones formativas favorece el aprendizaje y la retención de la información, además de proveer a los estudiantes de un mejor procesamiento cognoscitivo de los aspectos particulares de los ítems planteados en las evaluaciones. Se puede afirmar entonces que, el proveer a los estudiantes de pruebas de práctica constituye un recurso pertinente y simple de implementar como apoyo al estudio independiente.

También es posible relacionar el estudio independiente con la investigación de Ibabe y Jaureguizar (2007), en la cual se analizó la efectividad del uso de pruebas de autoevaluación interactivas en línea. La correlación que se obtuvo fue que mientras más pruebas formativas en línea realizaron los estudiantes, mayor fue la nota final que obtuvieron en el curso. En un estudio similar, Karagiani (2012) encontró que el uso de pruebas formativas diseñadas con una herramienta de autor mejoró el rendimiento académico de sus alumnos y contribuyó a cambiar la actitud negativa que tenían hacia la evaluación. Consiguientemente, las conclusiones obtenidas apuntan a que posiblemente no se haya aprovechado el máximo potencial de la evaluación formativa en la educación. Quizás no sea necesario diseñar estrategias didácticas completamente nuevas, sino redescubrir que potenciales ocultos tienen los enfoques tradicionales y remozarlos con las nuevas alternativas que brindan los recursos tecnológicos, tales como las herramientas de autor.

\section{Metodología}

\section{Descripción de la estrategia didáctica}

Para el desarrollo de la estrategia didáctica, el docente optó por la herramienta de autor "Hot Potatoes" con el fin de crear pruebas de práctica para sus estudiantes. Estas prácticas fueron adaptadas de evaluaciones utilizadas en años anteriores en el curso. Las pruebas se pusieron a disposición de los estudiantes en línea y contenían una lectura en formato PDF y una seria de ejercicios en formato HTML para analizarla. En total se diseñaron diez pruebas (dos como práctica para cada prueba corta del curso y una para cada examen). Las pruebas 
de práctica se estructuraron en cuatro partes generales, las cuales fueron similares a las empleadas en las evaluaciones del curso. Cada una de las partes se describirá a continuación:

Parte I-Pre-lectura: En esta parte, se utilizó los aspectos más generales de la lectura, tales como la introducción, las ilustraciones y otros datos iniciales, para que los estudiantes analizaran posibles predicciones del contenido del texto antes de leerlo con detenimiento, por medio de ítems de falso y verdadero con la adición de una tercera categoría denominada "imposible de decir" para disminuir la posibilidad de adivinar la respuesta por parte de los estudiantes (Heaton, 1990).

Parte II-Durante la lectura: Aquí los estudiantes pusieron en práctica las diversas estrategias de lectura desarrolladas en el curso para analizar la totalidad de la lectura, como el empleo eficiente del diccionario, el uso del contexto para generar significado, el análisis morfológico de palabras, el análisis de claves tipográficas, la identificación de sinónimos y antónimos, entre otras, en las cuales se emplearon formatos de selección única, asocie, respuesta corta y complete de textos con palabras faltantes.

Parte III-Comprensión de lectura: En esta parte, se volvió a analizar la totalidad de la lectura más a profundidad para que los estudiantes valoraran su comprensión del texto. Entre los ejercicios incluidos aquí, se utilizaron ítems de falso, verdadero y no mencionado (Heaton, 1990), organización de ideas de manera cronológica con distractores que contenían ideas no mencionadas en el texto, las cuales debían marcarse como "no aplicables" (Heaton, 1990), preguntas de comprensión de lectura general, discriminación entre hechos y opiniones, análisis de inferencias, organización de esquemas, entre otros, en los cuales se emplearon los formatos de selección única, respuesta corta y asocie.

Parte IV-Post-lectura: En la última parte, se invitó a los estudiantes a reflexionar sobre lo leído y relacionarlo con temas afines al contenido de la lectura por medio de preguntas retóricas y el análisis crítico de imágenes y caricaturas pertinentes a la temática del texto. En esta parte el formato de ítem empleado fue el de reorganizar preguntas desordenadas y completar textos con palabras faltantes.

\section{Papel del estudiante}

El papel del estudiante en este proyecto fue el de un aprendiz crítico y responsable de su propio aprendizaje. Para el buen aprovechamiento de los materiales de práctica, el estudiante debió disponer de un tiempo de estudio independiente semanal como se detalla en el programa de curso. Es importante señalar aquí que, en un curso de naturaleza práctica como en el que se enmarcó este proyecto, el desarrollo de las destrezas de lectura en una lengua extranjera se alcanza por medio de la práctica continua con diferentes textos; no es de mucha ayuda dedicarse a repasar la antología del curso solamente. 


\section{Papel del docente}

La labor del docente en el proyecto comprendió dos áreas. En primera instancia, el docente realizó el planteamiento teórico y práctico del proyecto y diseñó las pruebas de práctica interactivas para sus estudiantes. También, el docente desempeñó un papel de guía, orientador y facilitador del desarrollo de las destrezas de lectura en inglés de sus estudiantes en clase. En horario lectivo, el docente brindó a los estudiantes nociones, conceptos y práctica básicos para el empleo de diversas estrategias de lectura para que desarrollaran su comprensión de textos escritos en inglés. El docente también les mostró a los estudiantes cómo utilizar las pruebas de práctica interactivas que él puso a disposición en su página web personal.

\section{Participantes}

Los participantes de este proyecto fueron un grupo de 28 estudiantes (19 mujeres y 9 hombres) con un rango de entre 17 y 52 años de edad. Los estudiantes cursaban una gama muy amplia de carreras, tales como informática, ingeniería química, psicología, archivística, medicina, música, administración de negocios y matemáticas. Además, todos se encontraban en momentos distintos del desarrollo de sus carreras, los cuales iban desde primer año hasta licenciatura. El docente y autor de este artículo cuenta con ocho años de experiencia en la enseñanza del inglés, en cuatro de los cuales se ha desempeñado como docente universitario a nivel de grado y posgrado. Específicamente, el docente, al momento de poner en práctica el proyecto, había impartido el curso LM-1030, Estrategias de Lectura en Inglés I (Para otras carreras), catorce veces.

\section{Instrumento utilizado}

Para valorar la efectividad de la estrategia didáctica utilizada, el docente diseñó un cuestionario con cinco preguntas, de las cuales dos eran cerradas y tres abiertas (ver Apéndice). El instrumento primero les solicitaba a los estudiantes indicar si habían hecho uso de las pruebas de práctica interactivas, las cuales estuvieron a disposición de los estudiantes en la página web del docente o por qué motivo(s) no las utilizaron. El cuestionario también incluyó preguntas sobre la opinión general de los estudiantes acerca de los materiales, sugerencias para mejorarlos y una pregunta específica sobre el apoyo al estudio independiente brindado por las pruebas de práctica interactivas. Antes de administrar el cuestionario, el docente lo compartió con otro colega para realizar una revisión del instrumento y verificar que estuviera redactado con claridad y no fuera ambiguo. 


\section{Procedimientos}

Para la aplicación de la estrategia didáctica, se decidió que lo más conveniente sería realizarla durante la mayor parte del segundo semestre de 2013. Por lo tanto, el proyecto inició su aplicación en la tercera semana de clases (del 27 al 30 de agosto) y finalizó en la décimo cuarta semana (del 12 al 15 de noviembre). Con al menos una semana de antelación a cada prueba a realizarse en el curso (cuatro pruebas cortas y dos exámenes), el docente subió a su página web personal las pruebas de práctica diseñadas con "Hot Potatoes," dos para cada prueba corta y una por cada examen. También, el docente informó a los estudiantes sobre la disponibilidad de los materiales en horario lectivo y les mostró como utilizarlos por medio de una computadora y un proyector multimedia. En la semana de finalización del proyecto, el docente aplicó el cuestionario impreso a los estudiantes en horario lectivo y posteriormente realizó el análisis de los datos obtenidos.

\section{Consideraciones éticas}

La participación de los estudiantes en el proyecto fue totalmente voluntaria y no se les ofreció ningún beneficio por su participación, tal como puntos extra en la nota final. La única ventaja que tuvieron los participantes fue contar con material de práctica adicional como apoyo en sus horas de estudio independiente.

\section{Análisis de datos}

Las respuestas emitidas por los estudiantes en las primeras dos preguntas del cuestionario se tabularon de manera básica, para lo cual se utilizaron números de modo aclaratorio, más no estadístico. Para procesar las respuestas emitidas en las preguntas 3, 4 y 5 del cuestionario, se empleó la técnica de la codificación (Fraenkel, Wallen y Hyun, 2012), para cual se generaron categorías de análisis a posteriori para agrupar la información. Finalmente, la categorización de los datos se analizó y se comparó con la teoría utilizada en el proyecto para obtener las conclusiones.

\section{Resultados y discusión}

Antes de proceder a presentar los resultados obtenidos en el cuestionario, es necesario hacer una aclaración. El grupo con el cual se llevó a cabo el proyecto estaba constituido por 28 estudiantes. Sin embargo, al momento de aplicar el instrumento de valoración, únicamente se pudo obtener las respuestas de 21 estudiantes por diversas razones que se describirán a continuación. Un estudiante abandonó el curso a mitad del período lectivo. Dos estudiantes fueron 
incapacitados hacia el final del ciclo lectivo debido a intervenciones médicas. Tres estudiantes asistieron de manera inconsistente durante el ciclo lectivo y no estuvieron presentes cuando se aplicó el instrumento y, finalmente, una estudiante completó el instrumento de manera errónea, ya que se refirió a las presentaciones multimedia utilizadas por el docente en horario lectivo y no a los materiales diseñados con "Hot Potatoes," por lo que la información suministrada no se pudo utilizar para el análisis de datos del proyecto.

\section{Valoración de la estrategia desde la perspectiva del estudiante}

De la totalidad de los 21 estudiantes que completaron el cuestionario, 19 de ellos manifestaron haber utilizado las pruebas de práctica durante el ciclo lectivo. Los otros dos estudiantes dijeron no haber utilizado los materiales, uno por falta de tiempo y el otro porque no le parecieron útiles y añadió que no tuvo la necesidad de revisarlas. A continuación, se presentará el análisis de las respuestas específicas emitidas por los estudiantes según las categorías de análisis generadas.

\section{Ventajas ofrecidas por “Hot Potatoes” según los estudiantes}

Del total de estudiantes que usaron las pruebas de práctica, dieciocho utilizaron palabras positivas para expresar su opinión acerca de los materiales, como se puede apreciar en la tabla 1. Únicamente uno de los estudiantes manifestó que las prácticas tenían un nivel de dificultad diferente al de las evaluaciones formales del curso. La tabla 1 muestra la trascripción de las 18 respuestas positivas, las cuales se pudieron agrupar bajo una misma categoría. Al examinar el contenido de la tabla 1 , es posible notar que una marcada mayoría de los estudiantes consideraron que los materiales fueron útiles, ya que les permitieron prepararse para las evaluaciones formales del curso. Esto se relaciona con las ideas expuestas por Martínez et al. (2013), en el sentido de los estudiantes deben ser partícipes de su propio aprendizaje y por Ortiz (2011), con referencia a que el autoaprendizaje es más beneficioso, porque permite que los estudiantes vayan más allá de lo que se imparte en una clase presencial.

\section{Tabla 1}

\section{Preparación para las evaluaciones formales del curso}

\footnotetext{
- Fueron muy útiles, ya que nos permitía practicar y así lograr entender de mejor manera la materia.

- Son bastante útiles para estudiar y practicar antes de cada prueba.

- Son de gran utilidad, ya que me permitieron prepararme para los quices y exámenes del curso por su gran similitud.

- Me parece que son muy útiles y de gran ayuda para el curso.
} 
- Sí, son útiles para practicar.

- Es de mucha utilidad para reforzar lo aprendido en clase.

- Que son útiles para practicar y sobre qué esperar en las pruebas.

- Muy útiles para volver a repasar la materia y para practicar para los quices y exámenes.

- Muy útiles. Ayudan mucho a la hora de estudiar.

- El que corrijan automáticamente la respuesta es muy útil para identificar dónde se está fallando.

- Bastante buenos.

- Me parecieron muy buenos, porque es una forma interactiva para realizar los ejercicios.

- Son bastante buenos y ayudan a complementar lo visto en la clase.

- Muy buenos, ayudan a tener una práctica adicional y las presentaciones de las clases pasadas.

- Fueron de gran ayuda para estudiar y repasar los temas vistos en clase, tanto para un examen como para quices.

- Explican de manera resumida la materia y ayuda a conocer y entender distintos ejercicios en los que la materia se puede presentar.

- Dan un gran aporte complementario para las evaluaciones. Además son muy fáciles de realizar y una gran ventaja es que muestran cuál es la respuesta correcta.

- Son completos y se asemejan a las prácticas hechas en clase.

Nota: Respuestas tomadas de la pregunta 3 del cuestionario (ver Apéndice).

Resulta interesante en este momento además mencionar una anécdota que ocurrió durante el desarrollo del proyecto. El docente sostuvo conversaciones informales con los estudiantes sobre el uso de la pruebas de práctica en el curso y surgió un tema recurrente, el cual se puede relacionar con la información que aquí se discute. Muchos estudiantes manifestaron que ningún otro profesor en la universidad brinda este tipo de material de práctica. Algunos estudiantes incluso dijeron que ciertos docentes parece que se guardan la materia con recelo para que a los estudiantes les sea más difícil realizar las evaluaciones de un curso. No obstante, para que el componente evaluativo de un proceso de enseñanza y aprendizaje sea justo (válido), el estudiante debe saber qué se va a evaluar y cómo se va a evaluar.

\section{Apoyo al estudio independiente}

$\mathrm{Al}$ consultarles a los estudiantes si los materiales fueron de utilidad en sus horas de estudio independiente, la mayoría de las respuestas pudieron agruparse en tres grandes áreas, tal y como se muestra en la tabla 2. Las respuestas indeterminadas se colocaron en la cuarta columna de la tabla. 
Tabla 2

Ventajas de los materiales para el estudio independiente

\begin{tabular}{|c|c|c|c|}
\hline $\begin{array}{l}\text { Correspondencia con } \\
\text { el contenido impar- } \\
\text { tido y evaluado }\end{array}$ & $\begin{array}{l}\text { Reforzamiento de } \\
\text { conceptos }\end{array}$ & $\begin{array}{c}\text { Facilidad del trabajo } \\
\text { virtual }\end{array}$ & $\begin{array}{c}\text { Respuestas } \\
\text { indeterminadas }\end{array}$ \\
\hline $\begin{array}{l}\text { - Sí, porque se aplica- } \\
\text { ban a lo estudiado en } \\
\text { clase. } \\
\text { - Sí, ya que me permi- } \\
\text { tió realizar prácticas } \\
\text { de la materia vista en } \\
\text { clase. } \\
\text { - Sí, porque en otros } \\
\text { cursos los profesores } \\
\text { no se preocupan por } \\
\text { dar práctica para po- } \\
\text { ner en función lo que } \\
\text { se estudia en clase. } \\
\text { - Ayuda a practicar lo } \\
\text { visto en clase y prepa- } \\
\text { rarse para las pruebas. } \\
\text { - Sí, claro. Servían de } \\
\text { práctica y eran una } \\
\text { gran guía para quices } \\
\text { y exámenes. } \\
\text { - Sí, porque ayudan } \\
\text { a tener prácticas con } \\
\text { contenido que el pro- } \\
\text { fesor quiere evaluar, lo } \\
\text { que ayuda a saber en } \\
\text { qué manera van a ser } \\
\text { las pruebas, el tipo de } \\
\text { preguntas y la dificul- } \\
\text { tad que pretende tener. } \\
\text { - Sí, especialmente } \\
\text { para prepararse a las } \\
\text { pruebas cortas y los } \\
\text { exámenes. }\end{array}$ & $\begin{array}{l}\text { - Sí, ayudan a la hora } \\
\text { de entender la idea } \\
\text { fundamental del pá- } \\
\text { rrafo. } \\
\text { - Sí claro, porque me } \\
\text { ayudaron a reforzar } \\
\text { ciertas debilidades. } \\
\text { - Claro que sí. Me } \\
\text { permitieron repasar } \\
\text { la materia y darme } \\
\text { cuenta de qué temas } \\
\text { es necesario reforzar } \\
\text { para la posterior eva- } \\
\text { luación. } \\
\text { - Claro que sí, me ayu- } \\
\text { daron bastante ya } \\
\text { que me reforzaron las } \\
\text { dudas que se me iban } \\
\text { presentando. } \\
\text { - Sí, porque ayuda a } \\
\text { ampliar conceptos y } \\
\text { mediante la práctica } \\
\text { permite tener y desa- } \\
\text { rrollar las habilidades } \\
\text { para la lectura del in- } \\
\text { glés. }\end{array}$ & $\begin{array}{l}\text { - Sí, porque se pudo } \\
\text { practicar de una for- } \\
\text { ma más interactiva. } \\
\text { - Sí, son para mí más } \\
\text { útiles que el folleto } \\
\text { porque al dar la res- } \\
\text { puesta de inmediato } \\
\text { uno puede mejorar. } \\
\text { - Sí, porque en mi } \\
\text { casa me concentro } 10 \\
\text { mil veces más que en } \\
\text { clase. Resolviendo } \\
\text { prácticas virtuales en } \\
\text { donde realmente en- } \\
\text { tiendo. } \\
\text { - Sí, ya que mis horas } \\
\text { de estudio se basa casi } \\
\text { en su totalidad en los } \\
\text { materiales digitales } \\
\text { - Sí, porque al no po- } \\
\text { der asistir a todas las } \\
\text { clases por motivo de } \\
\text { mi trabajo no me atra- } \\
\text { so con los temas. }\end{array}$ & $\begin{array}{l}\text { - Sí, para practicar. } \\
\text { - En algunos casos, sí } \\
\text { útiles. }\end{array}$ \\
\hline
\end{tabular}

Nota: Respuestas tomadas de la pregunta 5 del cuestionario (ver Apéndice).

Las respuestas emitidas en la pregunta cinco del instrumento de valoración evidencian la validez y utilidad de las pruebas de práctica como estrategia de estudio (Dunlosky, Rawson, Marsh, Nathan, y Willingham, 2013), ya que son de gran utilidad al estudiante al comprender el planteamiento de ciertos ejercicios y poder reforzar individualmente aquellos conceptos que no le han quedado claros. Esto, en la opinión del autor de este artículo, constituye un tipo de práctica 
más real e incluso más justa. Además, el hecho de que los materiales ofrecieran realimentación inmediata fue un gran apoyo al estudio independiente de los estudiantes, ya que mejora el rendimiento general de los estudiantes (Karagiani, 2012; Ibabe y Jaureguizar, 2007). Asimismo, al finalizar cada ejercicio en "Hot Potatoes", se indican cuáles respuestas no son correctas y el estudiante recibe una nota. El docente ha podido observar algo positivo al respecto. A pesar de que el componente cuantitativo o de medición no es lo más importante en un proceso de evaluación de los aprendizajes, nuestra (in)cultura evaluativa nos hace querer ver números siempre. Tal compulsión por los números parece ser canalizada en una dirección correcta al utilizar "Hot Potatoes." Cuando un estudiante termina un ejercicio y recibe su nota y no es de su agrado, el estudiante generalmente vuelve a realizar el ejercicio (varias veces) hasta obtener una mejor puntuación, lo cual le estudiante moderar su aprendizaje y avanzar a su propio ritmo (Níkleva y López, 2012). El estudiante se ve forzado a revisar las respuestas que emitió previamente y a cuestionarse por qué no fueron correctas. También, al trabajar individualmente, se reduce el temor de parecer incapaz ante los demás; el estudiante puede concentrarse en su propio aprendizaje sin este tipo de cohibición.

\section{Sugerencias para mejorar los materiales}

En el apartado de sugerencias para mejorar los materiales (pregunta 4 del cuestionario, ver Apéndice), únicamente seis estudiantes señalaron puntos por corregir. Dos de ellos consideraron que se debe mejorar el aspecto de los ejercicios. Tres de ellos dijeron que debería haber más práctica y uno escribió que el formato a veces era diferente al utilizado en las evaluaciones formales del curso. En general, la apariencia de las actividades se puede cambiar con relativa facilidad, ya que "Hot Potatoes" permite que se modifiquen una gran variedad de características de las actividades, como los son el tipo y color de fuente, el color de los fondos de pantalla y cuadros de texto, sin dejar de lado la posibilidad de insertar imágenes en formatos tales como JPG y GIF. En cuanto al planteamiento de los ítems, sería necesario indagar más para saber cuáles ejercicios podrían plantearse de una manera más afín al formato de las evaluaciones formales del curso o por qué razón el estudiante consideró que existió una diferencia.

\section{Valoración de la estrategia desde la perspectiva del docente}

El diseño de pruebas de práctica por medio del uso de la herramienta "Hot Potatoes" le permitió al docente crear materiales para sus estudiantes de una manera fácil y relativamente rápida. Después de meditar sobre el mejor formato para presentar los contenidos a repasar en cada práctica y familiarizarse con las plantillas de diseño de actividades, el diseño de estas no tomó mucho tiempo. Además, luego de revisar el producto final, si de detectaba errores en la redacción, formulación o apariencia general de los ejercicios, resultó muy fácil realizar 
los cambios necesarios. También, utilizar una página web de acceso libre fue muy práctico a la hora de subir los materiales para los estudiantes y ponerlos a su disposición. En general, "Hot Potatoes" tiene una interfaz totalmente amigable con el usuario y como se mencionó anteriormente, posee bastantes características de apariencia que se pueden modificar a gusto del usuario y no existe un límite en la cantidad de texto o preguntas que se puedan ingresar en un mismo ejercicio. Otras herramientas de autor no ofrecen tales características.

\section{Conclusiones}

Tomando en cuenta la pregunta generadora “¿Es posible apoyar el estudio independiente de los estudiantes del curso LM-1030 por medio del uso de pruebas de práctica diseñadas con la herramienta "Hot Potatoes" y, de ser así, qué ventajas les ofrece a los estudiantes?", que se planteó para el proyecto, el apoyo al estudio independiente en los estudiantes por medio de pruebas de práctica diseñadas con "Hot Potatoes" no solo es posible, sino que también les ofrece a los estudiantes tres ventajas principales:

- Primero, los materiales creados les permiten a los estudiantes prepararse para las evaluaciones formales del curso y familiarizarse con posibles planteamientos de ítems en las evaluaciones, algo que puede constituir una limitante si los estudiantes desconocen la manera exacta en la cual se les va a evaluar.

- Segundo, al trabajar individualmente y sin temor a cometer errores frente a otras personas, cada estudiante puede ir a su propio ritmo, repasar contenidos y profundizar aquellos en los cuales aún tenga dudas.

- Tercero, el trabajo en línea y con materiales interactivos permite que los estudiantes no se queden únicamente con lo que reciben en clase, lo cual propicia que su formación académica continúe en otros entornos y se refuerce.

A continuación, el docente desea referirse en específico a las bondades, limitaciones y proyecciones de uso de "Hot Potatoes."

\section{Bondades}

"Hot Potatoes" es un programa muy versátil y simple de utilizar, lo que permite diseñar materiales y actividades rápidamente. Además, los materiales se pueden utilizar en línea o sin conexión a Internet si se han descargado con anterioridad. También, al trabajar en formato digital, es muy práctico volver a realizar una actividad con sólo cargar la página de nuevo, lo cual motiva al estudiante a realizar las actividades varias veces hasta obtener el resultado deseado y a trabajar a su propio ritmo. Esto es algo que no va a ocurrir en la mayoría de 
los casos si se trabaja con materiales impresos (Zúñiga, y Seravalli, 2014), lo que, a criterio del docente, apoya grandemente el proceso del estudio independiente.

\section{Limitaciones}

El uso de pruebas de práctica diseñadas con "Hot Potatoes" ofrece diversas ventajas. No obstante, esta modalidad de trabajo también supone ciertas limitaciones. El programa no podrá jamás sustituir a un docente a la hora de leer las diversas respuestas redactadas por un estudiante, por lo que no es factible plantear preguntas abiertas o ítems en los cuales el estudiante justifique una respuesta. Sólo es posible predeterminar ciertas respuestas correctas dentro de lo que permite el programa y el tipo de ejercicio, lo cual quiere decir que si hay ciertas respuestas que podrían expresarse de diferentes maneras y, si dichas maneras no se han considerado e ingresado en el programa, este no las reconocerá (Zúñiga, y Seravalli, 2014). Por esta misma razón, hay ciertos ejercicios que deben adaptarse de manera que se pueda trabajar con ellos en "Hot Potatoes," lo que hace que ciertos ítems se planteen de una manera ligeramente diferente a la de las evaluaciones del curso y podría provocar que el estudiante tenga dudas sobre como contestarlos, por ejemplo, que se utilice el formato de respuesta corta en lugar de selección única. La limitante anterior podría estar relacionada con la recomendación que brindó un estudiante en este sentido. Por otro lado, las prácticas digitales diseñadas para este proyecto venían en una carpeta comprimida, misma que el estudiante debía descargar y descomprimir para poder trabajar, por lo que el no tener el programa adecuado para este propósito o desconocer su funcionamiento podría ser un obstáculo.

\section{Proyecciones de uso}

"Hot Potatoes" permite el desarrollo de pruebas y materiales de práctica para una gama muy amplia de temáticas y contextos educativos. En sí, el uso de este programa informático no representa ningún reto para el docente. El verdadero reto yace en reflexionar sobre cuál es la manera más apropiada para hacer uso de él como apoyo o complemento para un curso y cuáles son los contenidos que se prestan más para crear recursos didácticos interactivos. Luego de tener estas consideraciones en mente, técnicamente no existe ningún contexto educativo en el cual un docente no pueda incorporar a "Hot Potatoes" en su repertorio de recursos didácticos. En el proyecto desarrollado, se utilizó "Hot Potatoes" para apoyar la práctica de la lectura en inglés. No obstante, esta herramienta es útil también para desarrollar ejercicios de gramática, escritura, comprensión auditiva, pronunciación y vocabulario, en el contexto específico de la enseñanza de idiomas, pero como se dijo anteriormente, puede ser utilizado en otros ámbitos educativos y cursos. 


\section{Bibliografía}

Díaz, F. y Muriá, I. (enero-junio, 1998). El desarrollo de habilidades cognoscitivas para promover el estudio independiente. Revista Electrónica Tecnología y Comunicación Educativas, 12(27) 17-27. Recuperado de http://investigacion.ilce.edu.mx/panel_control/doc/tecycomeduno27.pdf

Dudeney, G. y Hockly, N. (2008). How to Teach English with Technology. Harlow, Essex: Pearson Education.

Dunlosky, J., Rawson, K. A., Marsh, E. J., Nathan, M. J. y Willingham, D. T. (2013). Improving Students' Learning with Effective Learning Techniques: Promising Directions from Cognitive and Educational Psychology. Psychological Science in the Public Interest, 14(1), 4-58. doi: 10.1177/1529100612453266

Erben, T., Ban, R. y Castañeda, M. (2009). Teaching English Language Learners Through Technology. New York: Routlege. Recuperado de http://instek. llehuy.com/uploads/8/2/7/1/8271466/01_teaching.english.language.learners. through.technology2009.pdf

Fraenkel, J. C., Wallen, N. E. y Hyun, H. H. (2012). How to design and evaluate research in education ( ${ }^{\text {th }}$ ed.). New York: McGraw Hill.

Frei, S., Gammill, A. y Irons, S. (2007). Integrating Technology Into the Curriculum. Huntington Beach, CA: Shell Education. Recuperado de http://gec.kmu. edu.tw/ lc/ecorner/eBook/Integrating\%20Technology\%20Into\%20the\%20 Curriculum.pdf

Galarza, L. (1996). El estudio independiente en una visión sistémica de la educación a distancia. En P. Ávila y C. Morales (Eds.), Estudio independiente (pp. 21-35). México: ILCE-OEA-PROMESUP.

Half-Baked Software Inc. (2013). Hot Potatoes home page. Recuperado de http:// hotpot.uvic.ca/index.php

Half-Baked Software Inc. (2009). Hot Potatoes version 6. Recuperado de http:// www.halfbakedsoftware.com/hot_pot.php

Heaton, J. B. (1990). Writing English language tests. New York: Longman.

Ibabe, I. y Jaureguizar, J. (2007). Auto-evaluación a través de Internet: Variables metacognitivas y rendimiento académico. Revista Latinoamericana de Tecnología Educativa, 6(2), 59-75. Recuperado de http://campusvirtual.unex. es/revistas/index.php/relatec/article/view/319/301

Karagiani, E. (febrero, 2012). Employing Computer Assisted Assessment (CAA) to Facilitate Formative Assessment in the State Secondary School: A Case Study. Research Papers in Language Teaching and Learning, 3(1), 252-268. Recuperado de http://rpltl.eap.gr/images/2012/03-01-252-Karagianni.pdf

Níkleva, D. G. y López, M. (2012). Competencia digital y herramientas de autor en la didáctica de las lenguas. Tejuelo, 13, 123-140. Recuperado de http:// dialnet.unirioja.es/descarga/articulo/3804445.pdf

Martín, M. Á. (2004). Software de autor y estilos de aprendizaje. Didáctica (Lengua y Literatura), 16, 105-116. Recuperado de http://revistas.ucm.es/index. php/DIDA/article/view/DIDA0404110105A/19344 
Martínez, E., Martínez, M., Moyotl, E. S., Murguia, V. y Oliveros, I. M. G. (2013). El estudio independiente desde una visión colaborativa. Recuperado de http:// imagpeberosmont.blogspot.com/2013/05/el-estudio-independiente-desdeuna.html

Ortiz, M. (2011). La importancia del estudio independiente y su implicación en la administración del tiempo. Recuperado de http://www.cuaed.unam.mx/encuentro_2011/manuelortiz.pdf

Zúñiga, J. P. y Seravalli, G. (2014). Considering the use of Hot Potatoes in reading comprehension, autonomy in TEFL, and learning styles. Revista de Lenguas Modernas, 20, 309-321. Recuperado de http://revistas.ucr.ac.cr/index. $\mathrm{php} / \mathrm{rlm} /$ article/view/15068/14380

\section{Apéndice}

\section{Valoración de los materiales digitales interactivos provistos por el profesor}

Instrucciones: El presente instrumento tiene como objetivo sistematizar las opiniones de los estudiantes acerca de los materiales digitales interactivos que su profesor de curso puso a su disposición en Internet. Se le solicita responder de la manera más concisa y honesta las siguientes preguntas.

\section{Uso de los materiales}

1. ¿Utilizó durante este ciclo lectivo los materiales digitales interactivos que su profesor puso a su disposición en Internet? Marque con una equis (X).

( ) Sí (Pase a la pregunta 3)

( ) No (Pase a la pregunta 2)

2. ¿Por qué razón(es) no utilizó estos materiales? Marque con una equis todas las opciones que apliquen.

( ) No sabía que estaban disponibles.

( ) No le parecieron útiles. ¿Por qué?

( ) Tuvo problemas técnicos. Especifique:

() Otro Especifique:

Si contestó la pregunta 2 , este es el fin de este instrumento.

\section{Opinión sobre los materiales}

3. ¿Qué opina sobre los materiales digitales interactivos que su profesor puso a su disposición en Internet? 
4. ¿Tiene alguna sugerencia para mejorar estos materiales?

\section{Apoyo al estudio independiente}

5. ¿Considera que los materiales digitales interactivos que su profesor puso a su disposición en Internet fueron de utilidad en sus horas de estudio independiente para el curso? Explique. 